See discussions, stats, and author profiles for this publication at: https://www.researchgate.net/publication/333562123

\title{
A comparison of two reflectivity parametrizations in acoustic least-squares reverse time migration
}

Article in Exploration Geophysics · May 2019

DOI: 10.1080/08123985.2019.1682459

CITATIONS

2 authors:

0

Yongming Lu

Southern University of Science and Technology

9 PUBLICATIONS 6 CITATIONS

SEE PROFILE

Some of the authors of this publication are also working on these related projects:

Adaptive, unstructured-mesh-based acoustic/elastic reverse time migration and its GPU acceleration View project

Project $\quad$ Acoustic/Elastic LSRTM View project

READS

106

Qiancheng Liu

Princeton University

33 PUblicATIONS 58 CITATIONS

SEE PROFILE 


\title{
A comparison of two reflectivity parametrizations in
} acoustic least-squares reverse time migration

\author{
Yongming $\mathrm{Lu}^{1,2}$,Qiancheng $\mathrm{Liu}^{3 *}$ \\ ${ }^{1}$ Department of Earth and Space Sciences, Southern University of Science and Technology, \\ Shenzhen 518055, China. \\ ${ }^{2}$ School of Earth and Space Sciences, University of Science and Technology of China, Hefei, \\ Anhui 230026, China. \\ ${ }^{3}$ King Abdullah University of Science and Technology, Thuwal, 23955, Saudi Arabia. \\ ${ }^{*}$ Corresponding author \\ Email: qiancheng.liu@kaust.edu.sa
}

(May 29, 2019)

Running head: LSRTM

\begin{abstract}
In comparison with conventional reverse time migration (RTM), least-squares reverse RTM can improve imaging resolution and compensate irregular illumination caused by acquisition geometry and complex structures. Since proposed, as an advanced version of RTM, it has been applied a lot to improve resolution and balance amplitude in imaging. Generally, in LSRTM, there are two kinds of LSRTM reflectivity models: velocity perturbation related reflectivity model and normal-incidence reflection coefficient related reflectivity model. Each has its specific physical meaning and provides different inverted results. In this paper, we first give a brief review about the two different definitions. Then, we compare the differences of these two
\end{abstract}


methods, and build a mathematical relationship. In the definition related to reflection coefficient model we rescale the defined reflectivity with background velocity. Also, in source wavefield reconstruction, we use an effective trick by moving file pointer to fetch data from disk to reduce the memory cost. Finally, we test these two LSRTM schemes using the Marmousi model. We observe that the two inverted reflectivities are different, although they both image the subsurface discontinuities well. We furthermore extract traces from the inversion results and compare them with the ture reflectivity models, respectively, to verify the physical definitions of them.

Keywords: Acoustic, Least squares, Reverse-time, Migration 


\section{INTRODUCTION}

Reverse-time migration (RTM) is a power tool for imaging complex structures. However, it acts as the adjoint operator (Claerbout 1992), which is not a good approximation for the inverse operator. The advantage of Least-squares reverse time migration (LSRTM) to RTM is more significant for complex structures, and records with irregular acquisition aperture and limited frequency band. LSRTM can be used to approach the pseudoinverse operator.

Lailly (1983) introduced the concept of least-squares migration (LSM). Afterward, LSM has been extended to Kirchhoff migration (Schuster 1993; Nemeth et al. 1999), phase-shift migration (Kuehl and Sacchi 2002; Clapp et al. 2005), one-way wave equation (Wang et al. 2005), gaussian beam migration (Yuan et al. 2015; Yang et al. 2018; Yang and Zhu 2018) and is now on RTM. As an approach to reflectivity inversion, LSRTM has been discussed in a series of papers. Wong et al. (2011) used LSRTM to image the ocean-bottom data. A plane-wave source is used by Dai and Schuster (2013) to increase the computation efficiency of LSRTM. Tan and Huang (2014) numerically illustrated that it is crucial to update source wavefields to image steep dips. Dutta and Schuster (2014) took Q-attention in consideration when performing LSRTM. Zhang et al. (2015) introduced a practical LSRTM procedure based on a cross-correlative objective function. Wong et al. (2015) increased the LSRTM illumination by jointly using the reflection and free-surface-multiple data. Yao (2016) formulated LSRTM with a generalized matrix form. Liu et al. (2016b) improved the methods in Zhang et al. (2015) by using an efficient step-length formula. Liu (2016) and Liu et al. (2016a) showed that the elimination of redundant 
source effects can speed up the convergence behavior of LSRTM. Liu et al. (2017b) proposed a prestack approach for the methods in Zhang et al. (2015). Liu and Peter (2018) proposed to obtain an one-step LSRTM imaging by using Wiener filter in the data domain.

Because the pseudo-inverse operator is too expensive to compute and the inverse Hessian is too expensive to compute and store, we choose iterative inversion to approach the pseudoinverse operator. Generally, iterative LSRTM includes three steps: reverse time demigration (RTDM), reverse time migration (RTM), and searching direction estimation. RTDM based on Born approximation is responsible for the predicted data. RTM is the well-known adjoint operator (Plessix and Mulder 2004), in which the gradient is produced by cross-correlating the source wavefield with the residual wavefield, which are computed using the differences of observe and predicted data as adjoint sources. The gradient will be used to estimate the searching direction using some iterative optimization algorithms, such as conjugate gradient. To calculate the step length, we use a numerical line search approach as in the quasi-linear method to speed the convergence.

Here we focus on the constant-density acoustic wave equation based LSRTM. Generally, there are two kinds of workflows. Dai and Schuster (2013) used velocity perturbation as reflectivity model, which could be taken as the classic definition in Tarantola (1984). Zhang et al. (2015) used the normal-incidence reflection coefficients as reflectivity model, which is closely associated with the classical crosscorrelation imaging condition (Claerbout 1992). Under these two different reflectivity definitions in RTDM, two types of RTM gradients are produced, leading to 
two inverted results in different detailed appearances. In this paper, we compare the differences of these two LSRTM schemes and build a relationship between them. We find that an additional scaling of the background velocity is required in defining the reflection coefficient related reflectivity.

In addition, we also try to alleviate the expensive boundary storage of source wavefield with a small trick by flexibly moving the file pointer with fseek. Here, fseek is a $\mathrm{C}$ function belonging to the ANSI C standard library, and included in the file stdio.h. Its purpose is to change the file position indicator for the specified stream. Rather than storing the whole source wavefield history on the disk, which will result in a heavy communication burden, we output the boundary of source wavefields to disk. Then, during the source wavefield reconstruction, we read the stored boundary into memory at each time-marching step. According to our numerical test, this trick is simple but practical, and could also be used in receiver wavefield propagation. This trick is also useful in accelerating RTM on GPU, the global memory space of which is usually not as large as that of CPU.

The paper is arranged as following: First, we briefly review least-squares migration and two RTDMs with different reflectivity parameterizations. Then, we compare the two RTDMs and try to bridge the gap between them. Also, we evaluate the cost of LSRTM during numerical implementation, and try to alleviate the memory cost of source wavefield reconstruction using a small trick. Finally, our schemes are verified in numerical examples.

\section{METHODS}




\section{Review of least-squares migration}

Born modeling, which is the first-order approximation to full acoustic wave equation, can be written as

$$
\mathrm{Lm}=\mathrm{d},
$$

where $\mathbf{L}$ is the forward operator associated with a specific configuration of survey geometry, velocity model, reflectivity model $\mathbf{m}$ and source wavelet, and $\mathbf{d}$ is the observed data. In seismic migration, usually we only have $\mathbf{d}$ and migration velocity at hand, attempting to image the subsurface structures. Because of unbalanced illumination and limited acquisition aperture, the output stacked imaging profile is inferior compared with the true reflectivity model. To compensate the limited resolution and unbalanced amplitudes in RTM images, we solve an inverse problem for reflectivity model. The misfit function can be expressed as

$$
E(\mathbf{m})=\frac{1}{2}\|\mathbf{L m}-\mathbf{d}\|_{2}^{2},
$$

the pseudo solution that satisfies $\frac{d E(\mathbf{m})}{d \mathbf{m}}=0$ can be expressed as

$$
\mathbf{L}^{T} \mathbf{L m}=\mathbf{L}^{T} \mathbf{d}
$$

where $\mathbf{L}^{T}$ is the adjoint operator of $\mathbf{L}$. In conventional migration we have

$$
\mathbf{m}_{m i g}=\mathbf{L}^{T} \mathbf{d},
$$


from which we find that $\mathbf{L}^{T} \mathbf{L}$, the Hessian, acts as the blur operator on $\mathbf{m} . \mathbf{m}_{m i g}$ is the blurred version of $\mathbf{m}$. However, the Hessian matrix in practical problems is prohibitive to compute and store. As a proper solution, we choose iterative inversion using gradient optimization method, such as steepest descent or conjugate gradient method (Nemeth et al. 1999), to deblur the adjoint migration result $\mathbf{m}_{m i g}$ to approach the reflectivity model. Although Zhang et al. (2015) used a crosscorrelative objective function, the mathematical essence hidden behind their method could still be classified under the iterative inversion.

\section{Review of two reverse-time demigrations}

In the time and space domain, the acoustic wave equation with constant density is given by

$$
\left(\frac{1}{v(\mathbf{x})^{2}} \frac{\partial^{2}}{\partial t^{2}}-\nabla^{2}\right) p\left(\mathbf{x}, t ; \mathbf{x}_{S}\right)=s\left(t ; \mathbf{x}_{S}\right)
$$

where $v(\mathbf{x})$ is the velocity, $p\left(\mathbf{x}, t ; \mathbf{x}_{S}\right)$ is the pressure field excited by the source $s\left(t ; \mathbf{x}_{S}\right)$ at position $\mathbf{x}_{S}$. A true velocity model $v(\mathbf{x})$ contains a background $v_{0}(\mathbf{x})$ and a perturbation $\delta v(\mathbf{x})$. Expanding the velocity in a Taylor series and dropping quadratic terms:

$$
\frac{1}{\left(v_{0}(\mathbf{x})+\delta v(\mathbf{x})\right)^{2}} \approx \frac{1}{v_{0}(\mathbf{x})^{2}}-\frac{2 \delta v(\mathbf{x})}{v_{0}(\mathbf{x})^{3}}
$$


and substituting it into Equation 5 yields following equations:

$$
\left\{\begin{array}{l}
\left(\frac{1}{v_{0}(\mathbf{x})^{2}} \frac{\partial^{2}}{\partial t^{2}}-\nabla^{2}\right) p_{0}\left(\mathbf{x}, t ; \mathbf{x}_{S}\right)=s\left(t ; \mathbf{x}_{S}\right) \\
\left(\frac{1}{v_{0}(\mathbf{x})^{2}} \frac{\partial^{2}}{\partial t^{2}}-\nabla^{2}\right) \delta p\left(\mathbf{x}, t ; \mathbf{x}_{S}\right)=\frac{m(\mathbf{x})}{v_{0}(\mathbf{x})^{2}} \frac{\partial^{2} p_{0}\left(\mathbf{x}, t ; \mathbf{x}_{S}\right)}{\partial t^{2}},
\end{array}\right.
$$

Following Tromp et al. (2005), the reflectivity here is defined as $m_{\delta v}(\mathbf{x})=2 \delta v(\mathbf{x}) / v_{0}(\mathbf{x})$. Two modelings are needed in the demigration process: one for $p_{0}$, the other one following for $\delta p$. Then, the demigrated wavefield $\delta p$ driven by the product of $m(\mathbf{x})$ and $p_{0}$ can be obtained, during which the term $\frac{m_{\delta v}(\mathbf{x})}{v_{0}(\mathbf{x})^{2}} \frac{\partial^{2} p_{0}\left(\mathbf{x}, t ; \mathbf{x}_{S}\right)}{\partial t^{2}}$ acts as a right-hand-side (RHS) virtual source for the first-order equation. For simplification, we express the demigration process in LSRTM by a concise matrix form:Lm=d, where $\mathbf{L}$ represents the forward operator, $\mathbf{m}$ represents the reflectivity model, and $\mathbf{d}$ represents the predicted data.

Similarly, the demigration process, i.e., the forward operator, in Zhang et al. (2015) could be expressed as

$$
\left\{\begin{array}{l}
\left(\frac{1}{v_{0}(\mathbf{x})^{2}} \frac{\partial^{2}}{\partial t^{2}}-\nabla^{2}\right) p_{0}\left(\mathbf{x}, t ; \mathbf{x}_{S}\right)=s\left(t ; \mathbf{x}_{S}\right) \\
\left(\frac{1}{v_{0}(\mathbf{x})^{2}} \frac{\partial^{2}}{\partial t^{2}}-\nabla^{2}\right) \delta p\left(\mathbf{x}, t ; \mathbf{x}_{S}\right)=r(\mathbf{x}) \frac{\partial p_{0}\left(\mathbf{x}, t ; \mathbf{x}_{S}\right)}{\partial t}
\end{array}\right.
$$

in which $r(\mathbf{x})$ is reflection coefficient related model. If we ignore the amplitudeversus-angle (AVA) information and only use the stacked image in the inversion, $r(\mathbf{x})$ could be approximated with the normal-incidence reflection coefficient model. We could also express this demigration process in the compact matrix form: $\mathbf{L m}=\mathbf{d}$. The difference between the two Born modelings is that $m_{\delta v}(\mathbf{x})=2 \delta v(\mathbf{x}) / v_{0}(\mathbf{x})$ in 
the first one while $m_{r}(\mathbf{x})=r(\mathbf{x})$ in the second one.

From $\delta v(\mathbf{x})$ to $r(\mathbf{x})$

By giving insights into the physical definitions of reflection coefficient and velocity perturbation, we attempt to establish an approximate relationship between them. Since reflection data are usually acquired at near offsets, considering a simple local velocity contrast of $v_{1}$ and $v_{2}$ by using vertical rays and constant density assumption (Wu et al. 2015), the near normal-incident reflection coefficient $r_{12}$ (Aki and Richards 1980) reads

$$
r_{12} \approx \frac{\left(v_{2}-v_{1}\right)}{\left(v_{2}+v_{1}\right)}=\frac{\left(v_{0}+\delta v_{2}\right)-\left(v_{0}+\delta v_{1}\right)}{\left(v_{0}+\delta v_{2}\right)+\left(v_{0}+\delta v_{1}\right)} \approx \frac{\delta v_{2}-\delta v_{1}}{2 v_{0}}
$$

where $v_{0}$ denotes the homogeneous background velocity and $\delta v=v-v_{0}$ denotes the velocity perturbation. The perturbation $\delta v$ is negligible compared to the background $v_{0}$, because the Born approximation is valid in the case of small and weak perturbations (Virieux and Operto 2009). In Figure 1, we confirm this approximate relationship using a toy example. In Figure 1d, the theoretical value should be 0.0909 while our estimated value is 0.0879 .

The first-order spatial derivative form Equation 9 reads

$$
2 v_{0} r_{12}=d x \frac{\delta v_{2}-\delta v_{1}}{d x}=\frac{\partial \delta v}{\partial x}
$$

For an evenly spatial sampling, $d x$ is a constant so we drop it from the right-hand 
side (RHS). Then, a generalized form of Equation 10 can be represented as

$$
2 v_{0}(\mathbf{x}) r(\mathbf{x})=\frac{\partial \delta v(\mathbf{x})}{\partial l}=\frac{\partial \delta v(\mathbf{x})}{\partial \mathbf{x}} \cdot \mathbf{n}(\mathbf{x})
$$

where $\mathbf{x}$ represents the spatial coordinates, $\mathbf{n}(\mathbf{x})$ represents the directional vector normal to the reflector. Equation 11 indicates an approximate relationship between reflection coefficient $r(\mathbf{x})$ and velocity perturbation $\delta v(\mathbf{x})$ in the near normal-incident case under Born approximation.

In frequency domain, the spatial derivative operator $\partial / \partial l$ could be expressed as $i w / v$, so we rewrite Equation 11 as

$$
r(\mathbf{x})=\frac{i w}{2 v_{0}(\mathbf{x})^{2}} \delta v(\mathbf{x})
$$

Plugging Equation 12 back to Equation 7 yields a new form of Equation 8 as following:

$$
\left\{\begin{array}{l}
\left(\frac{1}{v_{0}(\mathbf{x})^{2}} \frac{\partial^{2}}{\partial t^{2}}-\nabla^{2}\right) p_{0}\left(\mathbf{x}, t ; \mathbf{x}_{S}\right)=s\left(t ; \mathbf{x}_{S}\right) \\
\left(\frac{1}{v_{0}(\mathbf{x})^{2}} \frac{\partial^{2}}{\partial t^{2}}-\nabla^{2}\right) \delta p\left(\mathbf{x}, t ; \mathbf{x}_{S}\right)=\frac{r(\mathbf{x})}{v_{0}(\mathbf{x})} \frac{\partial p_{0}\left(\mathbf{x}, t ; \mathbf{x}_{S}\right)}{\partial t}
\end{array}\right.
$$

we find that in the new definition above $r(\mathbf{x})$ is spatially divided by an extra scaling factor $v_{0}(\mathbf{x})$. In our new LSRTM, the $r(\mathbf{x})$ related model $m_{r}(\mathbf{x})$ is expressed as $m_{r}(\mathbf{x})=r(\mathbf{x}) / v_{0}(\mathbf{x})$ 


\section{Reverse-time migrations(RTMs)}

We already have the forward operators $\mathbf{L}$ with respect to $m_{\delta v}(\mathbf{x})$ and $m_{r}(\mathbf{x})$ in Equations 7 and 13, respectively. In this section we will turn to seek $\mathbf{L}^{T}$, the adjoint operator of $\mathbf{L} . \mathbf{L}^{T}$, could also be called the RTM operator. According to adjoint method (Plessix and Mulder 2004; Dai and Schuster 2013), the specific process about how to get the adjoint operators is formulated in Appendix A. When the reflectivity model is defined as $\delta v(\mathbf{x}) / v_{0}(\mathbf{x})$, the gradient could be obtained by applying the following imaging condition

$$
m_{\delta v}(\mathbf{x})=\int_{0}^{T}-\frac{\partial^{2} p_{0}}{v_{0}^{2} \partial t^{2}} \hat{p} d t
$$

where $p_{0}$ is the forward propagated source wavefied, and $\hat{p}$ is the adjoint wavefield governed by the following equation

$$
\left(\frac{\partial^{2}}{v_{0}(\mathbf{x})^{2} \partial t^{2}}-\nabla^{2}\right) \hat{p}\left(\mathbf{x}, t ; \mathbf{x}_{S}\right)=\Delta d\left(\mathbf{x}_{R}, t ; \mathbf{x}_{S}\right)
$$

in which $\Delta d\left(\mathbf{x}_{R}, t ; \mathbf{x}_{S}\right)$ denotes the data residual at receiver $\mathbf{x}_{R}$. Similarly, when re-

flectivity model is defined as $r(\mathbf{x}) / v_{0}(\mathbf{x})$, we have the imaging condition as following

$$
m_{r}(\mathbf{x})=\int_{0}^{T}-\frac{\partial p_{0}}{\partial t} \hat{p} d t
$$


in which the adjoint wavefield $\hat{p}$ is governed by Equation 15 as well. After rearranging the term $-\frac{\partial}{\partial t}$ to $\Delta d\left(\mathbf{x}_{R}, t ; \mathbf{x}_{S}\right)$, we can get a new form of Equation 16 as following

$$
m_{r}(\mathbf{x})=\int_{0}^{T} p_{0} \hat{p} d t
$$

in which the adjoint wavefield $\hat{p}$ is governed by

$$
\left(\frac{\partial^{2}}{v_{0}(\mathbf{x})^{2} \partial t^{2}}-\nabla^{2}\right) \hat{p}\left(\mathbf{x}, t ; \mathbf{x}_{S}\right)=-\frac{\partial \Delta d\left(\mathbf{x}_{R}, t ; \mathbf{x}_{S}\right)}{\partial t}
$$

Now the $\mathbf{L}^{T}$ operators composed of Equations 14 and 15, Equations 17 and 18 nearly have the same forms with those in Dai and Schuster (2013), Zhang et al. (2015), respectively.

\section{Numerical implementation and Computation \& I/O cost}

No matter what kind of parameterization is chosen for the two definitions of reflectivity, we could use the linearized least-squares inversion to invert for the reflectivity images (Lailly 1983). In iterative LSRTM using conjugate gradient method (Nemeth et al. 1999), two RTDMs and one RTM are required: one RTDM to generate the predicted data to match the observed data, one RTM to produce a gradient with respect to the data residual, and the other RTDM for estimating a step length to scale the searching direction, for example, when using conjugate gradient method.

Each RTDM needs two modelings: one forward-propagated source wavefield to drive the other demigated wavefield. In conventional cross-correlated RTM, an efficient strategy to reconstruct the source wavefield is to firstly store the forwardpropagated source wavefield at boundaries, and then back-propagate the values 
from boundaries. At the same time, the receiver wavefield is backward propagated to cross-correlate with the reconstructed source wavefield to produce a gradient by using the imaging condition. That is, three modelings are required during this process.

In LSRTM, because the background velocity $v_{0}(\mathbf{x})$ stays invariant over linearized iterations, the source wavefield $p_{0}\left(\mathbf{x}, t ; \mathbf{x}_{S}\right)$ in RTM is just the source wavefield we computed in RTDM. Therefore, $p_{0}\left(\mathbf{x}, t ; \mathbf{x}_{S}\right)$ stored at boundaries could be written into memory only once, and then it could repeatedly serve for all the source wavefield reconstruction. It is well known that even if we only keep the value of $p_{0}\left(\mathbf{x}, t ; \mathbf{x}_{S}\right)$ at boundaries to reconstruct the source wavefield, the storage cost is high. Assuming the boundary consists of $\mathbf{n b}$ points and the number of time step is $\mathbf{n t}$. Conventionally a big array at size $\mathbf{n b} * \mathbf{n t}$ is required. Sometimes this memory storage could become the main bottleneck that limits the application of RTM to practical problems in large sacle. Some authors propose to reduce this memory storage by interpolation (Liu et al. 2015), but surely in their method some additional computational cost will arise. Usually the disk capability is significantly much larger than the memory capability.

In recent years, the accessing bandwidth of storage media (hard disk) is growing broader and broader while its price is getting lower and lower. For example, the accessing bandwidth of solid-state disk (SSD) could be only ten times slower than that of computer memory. In this paper, to further reduce the storage cost, we put the boundary source wavefield in a data file on the disk, and then use fseek() to move the file pointer within the data file in a well-organized order to access the 
data. In this way, only a small array at size nb is enough, as shown in Figure 2. Some codes about how to use fseek are shown in Appendix B. We could adapt this method in receiver wavefield propagation as well. According to our experiences, while conserving the memory cost significantly, this strategy will not obviously slow down the performance of RTM. The additional computational cost is negligible compared with the total RTM cost.

In addition, we usually choose the inner boundaries adjacent to PML layers for the boundary storage of $p_{0}\left(\mathbf{x}, t ; \mathbf{x}_{S}\right)$. The stored values are used as boundary condition rather than fictitious sources during wavefield reconstruction. Therefore, we only compute the interested domain enclosed by PML layers, no computation inside PML layers is required. In this way we could slightly alleviate the expensive computational cost furthermore.

\section{NUMERICAL EXAMPLES}

\section{Two-layer model}

We first verify the two $m_{\delta v}(\mathbf{x})$ and $m_{r}(\mathbf{x})$ related reflectivity models using a two-layer model, as shown in Figure 3a. The model ranges from 0 to $3 \mathrm{~km}$ and the grid interval is $10 \mathrm{~m}$ in the $x$ - and $z$-directions; the reflection layer is inserted at a depth of 2 $\mathrm{km}$. The velocity ranges from 2 to $3 \mathrm{~km} / \mathrm{s}$. Figures $3 \mathrm{~b}$ and $3 \mathrm{~d}$ show the corrponding migrated images using the two related reflectivity models. After twenty iterations, iterative LSRTM drives the imaging to approach inverted results in Figures 3c and 3e. Figures $4 \mathrm{a}$ and $4 \mathrm{~b}$ show the extracting traces at location $1.5 \mathrm{~km}$ from Figures $3 \mathrm{c}$ and $3 \mathrm{e}$, respectively. We can see that the inverted two related reflectivity models 
of the two-layer model match well with Figures $1 \mathrm{c}$ and $1 \mathrm{~d}$. This numerical example demonstrates the performance of the two related reflectivity models.

\section{D Marmousi model}

In this section, we verify our method using the 2D Marmousi model. Performing an $\mathrm{O}\left(d t^{2}, d x^{4}\right)$ staggered-grid finite-difference scheme (Virieux 1986), the observed data is generated from the true velocity model in Figure 5a rather than the reflectivity plus the background velocity model, so no inversion crime is committed (Clément et al., 2001). 150 shots are excited evenly at a 50-m interval. The receiver data is acquired with a fixed-spread acquisition geometry. Each shot has 801 receivers with a $5 \mathrm{~m}$ interval to get the shot records. A Ricker wavelet with a center frequency of $20-\mathrm{Hz}$ is used here. The recording time is $4 \mathrm{~s}$. In our LSRTM, the modified zero-lag cross-correlation imaging condition (Liu et al. 2017a) is performed. Figures 5a and 5b show the true and the background velocities, respectively. Figure 6a shows the true reflectivity model related to velocity perturbation for reference is calculated as the difference between the true and smooth velocities using $m_{\delta v}(\mathbf{x})=\delta v(\mathbf{x}) / v_{0}(\mathbf{x})$. The other true reflectivity model related to normal incidence reflection coefficient shown in Figure 7a is computed from the true velocity model using vertical rays and constant density assumption. Here we define $m_{r}(\mathbf{x})=r(\mathbf{x}) / v_{0}(\mathbf{x})$.

In both inversions for $m_{\delta v}(\mathbf{x})$ and $m_{r}(\mathbf{x})$ we use conjugate gradient optimization scheme. We first migrate the observed data using the smooth model in Figure 5b. As shown in Figures $6 \mathrm{~b}$ and $7 \mathrm{~b}$, the migration profiles look imperfect due to the adjoint operator plus the band-limited source wavelet, the unbalanced source 
illumination and the limited receiver aperture. In RTM stage we use the trick of fseek for source wavefield reconstruction, numerical experience shows that this trick will only slow down the total computation time by less than $0.1 \%$ compared with the memory storage, while we could reduce the memory cost dramatically. For exmaple, given a source wavefield reconstruction simulation with 5000 time steps, if we input a slice of 500 slice per communication, the memory could be only $10 \%$ of the originally required memory. The same trick could also be used for receiver wavefield propagation. After several iterations, iterative LSRTM drives the imaging profiles to approach inverted results in Figures 6c and 7c, matching well with the true reflectivity models in Figures $6 \mathrm{a}$ and $7 \mathrm{a}$, respectively. To further illustrate the enhancement of imaging resolution, we compare the amplitude spectra of RTM image and two LSRTMs images in Figure 9. The convergence curves of two LSRTMs are shown in Figure 10, $m_{\delta v}(\mathbf{x})$ inversion in red and $m_{r}(\mathbf{x})$ inversion in blue. We notice that at the early stage the blue curve has a faster convergence speed while at the late stage the red curve has a better convergence rating. Our explanation to this is: compared with $m_{r}(\mathbf{x})$ inversion using imaging condition in Equation 16, $m_{\delta v}(\mathbf{x})$ inversion uses Equation 14 to seek for gradient, which implicitly has more high-wavenumber components at the early stage because of the additional $\frac{\partial}{\partial t}$ term; but on the physical essence of $m_{\delta v}(\mathbf{x})$, it has two big slide lobes around each reflector, as shown in Figure 1c, so that some small reflectivity details would be more or less masked by side-lobes of the main structure reflectivities nearby at the late inversion stage; $m_{r}(\mathbf{x})$ defines reflectivity as Delta function which will not suffer from extra big side lobes in physics. 
Two zoom-view groups taken from the inverted results and true reflectivity model are shown in Figure 8. For detailed investigation, we extract traces from inverted results and true reflectivity model at the location $\mathrm{X}=4 \mathrm{~km}$ in Figures $6 \mathrm{a}$ and $6 \mathrm{c}$, Figures 7a and 7c, respectively. In Figure 11, in each comparison group, the inverted trace matches well with its corresponding true model trace. This implies that the reflectivity definitions in $m_{\delta v}(\mathbf{x})=\delta v(\mathbf{x}) / v_{0}(\mathbf{x})$ and $m_{r}(\mathbf{x})=r(\mathbf{x}) / v_{0}(\mathbf{x})$ are basically correct, and two LSRTMs work well. Note that in the definition of $m_{r}(\mathbf{x})$, we scale the $r(\mathbf{x})$ with $1 / v_{0}(\mathbf{x})$.

\section{DISCUSSION}

To better understand the reflectivity model in least-squares reverse time migration, a comparison of two reflectivity parametrizations is proposed. The numerical examples show that both the velocity perturbation related reflectivity model $m_{\delta v}(\mathbf{x})$ and incidence reflection coefficient related reflectivity model $m_{r}(\mathbf{x})$ of LSRTM are effective to improve the image quality compared to the conventional RTM image. Here, we present more detailed illustrations about these two parametrizations.

As shown in Figure 10, we can notice the $m_{r}(\mathbf{x})$ related one converges a bit slower than the $m_{\delta v}(\mathbf{x})$ one. The reason behind this phenomenon is that the $m_{r}(\mathbf{x})$ related one is a further approximation to the $m_{\delta v}(\mathbf{x})$ related one. Also, in the $m_{r}(\mathbf{x})$ approximation, we neglect the AVA behaviors, which should be considered in the further research. When the data is generated from the true velocity model, the latter performs a bit better than the former. We also extract well-logs at location $4 \mathrm{~km}$ from the true reflectivity models and the inverted results in Figures 6 and 7, respectively. Figure 11 shows the comparisons, we can observe that the inverted 
results match well in terms of amplitude with the true well-logs. Note that Figure 11b presents more high-wavenumber components than Figure 11a. The reason is due to their physical definitions, $m_{\delta v}(\mathbf{x})$ has two big slide lobes around each reflector, as shown in Figure 1c, so that some small reflectivity details would be more or less masked by side-lobes of the main structure reflectivities nearby at the late inversion stage; $m_{r}(\mathbf{x})$ defines reflectivity as Delta function which will not suffer from extra big side lobes. Therefore, the $m_{\delta v}(\mathbf{x})$ model has better convergence behavior in term of misfit function curve, the $m_{r}(\mathbf{x})$ model has better spatial resolution due to its physical definition.

\section{CONCLUSIONS}

LSRTM is an effective alternative for obtaining high-quality images for RTM. In this paper, we build a connection between two reflectivity definitions in mathematics and physics, in which we find a scaling factor related to background velocity when defining normal incidence reflection coefficient related reflectivity model. We also evaluate the LSRTM computational cost and introduce a trick to reduce memory cost for source wavefield reconstruction. In the numerical example, we compare the inverted results and convergence speed between two LSRTMs. We find that $m_{\delta v}(\mathbf{x})$ inversion has a slightly faster convergence speed at the early stage while $m_{r}(\mathbf{x})$ inversion convergence curve finally catches up and reaches at a better rate. We explain their convergence behavior differences with their physical meanings and their corresponding gradients. Finally, we confirm our physical definitions by extracting traces from the inverted results and true reflectivity models, respectively.

\section{ACKNOWLEDGMENTS}


We are grateful to Associate editor Jianxiong Chen and three reviewers for very kind comments and helpful suggestions which greatly improved the quality of our paper. We thank the National Natural Science Fund of China (under grant 41330316) and China Postdoctoral Science Foundation (under grant 2019M652196) for supporting this work.

\section{APPENDIX A}

\section{FORWARD AND ADJOINT OPERATOR FOR $M(\mathrm{X})$ IN MATRIX FORM}

A compact matrix form of the forward operator denoted by Equation 7 could be expressed in frequency domain as

$$
\mathrm{Fp}=\mathrm{s}
$$

where

$$
\mathbf{F}=\left(\begin{array}{cc}
\frac{\partial^{2}}{v_{0}(\mathbf{x})^{2} \partial t^{2}}-\nabla^{2} & 0 \\
-\frac{m(\mathbf{x}) \partial^{2}}{v_{0}(\mathbf{x})^{2} \partial t^{2}} & \frac{\partial^{2}}{v_{0}(\mathbf{x})^{2} \partial t^{2}}-\nabla^{2}
\end{array}\right), \mathbf{p}=\left(\begin{array}{c}
p_{0}\left(\mathbf{x}, t ; \mathbf{x}_{S}\right) \\
\delta p\left(\mathbf{x}, t ; \mathbf{x}_{S}\right)
\end{array}\right), \mathbf{s}=\left(\begin{array}{c}
s\left(t ; \mathbf{x}_{S}\right) \\
0
\end{array}\right)
$$

in which $m(\mathbf{x})=\delta v(\mathbf{x}) / v_{0}(\mathbf{x})$ is the defined reflectivity, $v_{0}(\mathbf{x})$ is the background velocity or migration velocity, $p_{0}\left(\mathbf{x}, t ; \mathbf{x}_{S}\right)$ and $\delta p\left(\mathbf{x}, t ; \mathbf{x}_{S}\right)$ wavefield and perturbation wavefields excited by the source signal $s\left(t ; \mathbf{x}_{S}\right)$ at shot location $\mathbf{x}_{S}$. If we focus on the relationship between reflectivity $m(\mathbf{x})$ and the demigrated data $\delta p\left(\mathbf{x}_{R}, \omega ; \mathbf{x}_{S}\right)$ at 
$\mathbf{x}_{R}$, in least-squares sense, we could express the misfit function in data domain as

$$
\phi(\mathbf{m})=\frac{1}{2}\langle\mathbf{p}(\mathbf{m})-\mathbf{d}, \mathbf{p}(\mathbf{m})-\mathbf{d}\rangle
$$

where $\langle$,$\rangle is the dot-product operator, \mathbf{p}(\mathbf{m})$ denotes the predicted data and $\mathbf{d}$ denotes the observed data. The gradient of $\phi(\mathbf{m})$ with respect to $\mathbf{m}$ could be written as

$$
\frac{\partial \phi(\mathbf{m})}{\partial \mathbf{m}}=\left\langle\frac{\partial \mathbf{p}(\mathbf{m})}{\partial \mathbf{m}}, \mathbf{p}(\mathbf{m})-\mathbf{d}\right\rangle
$$

Taking the first-order derivative with respect to $\mathbf{m}$ in Equation A-1 yields

$$
\frac{\partial \mathbf{F}}{\partial \mathbf{m}} \mathbf{p}+\mathbf{F} \frac{\partial \mathbf{p}}{\partial \mathbf{m}}=\mathbf{0}
$$

Then we have

$$
\frac{\partial \mathbf{p}}{\partial \mathbf{m}}=-\mathbf{F}^{-1} \frac{\partial \mathbf{F}}{\partial \mathbf{m}} \mathbf{p}
$$

After substituting equation (A-6) back to Equation A-4 and rearranging terms we have

$$
\frac{\partial \phi(\mathbf{m})}{\partial \mathbf{m}}=-\left\langle\frac{\partial \mathbf{F}}{\partial \mathbf{m}} \mathbf{p},\left(\mathbf{F}^{-1}\right)^{\mathrm{T}}(\mathbf{p}-\mathbf{d})\right\rangle
$$

in time domain, we could directly use the transpose operator ()$^{\mathrm{T}}$ instead of the conjugate transpose operator ()$^{*}$, and have $\left(\mathbf{F}^{-1}\right)^{\mathrm{T}}=\left(\mathbf{F}^{\mathrm{T}}\right)^{-1}$. According to the 
adjoint method, we set $\hat{\mathbf{p}}=\left(\mathbf{F}^{\mathrm{T}}\right)^{-1}(\mathbf{p}-\mathbf{d})$, yielding

$$
\mathbf{F}^{\mathrm{T}} \hat{\mathbf{p}}=\mathbf{p}-\mathbf{d}
$$

in which the solution is $\hat{\mathbf{p}}$ called adjoint wavefield of data residual $\mathbf{p}-\mathbf{d}$. Assuming we only record the back scattering wavefield, we have $\mathbf{p}-\mathbf{d}=(0, \Delta d)^{\mathrm{T}}$. Then, in matrix form Equation A-8 could be expressed as

$$
\left(\begin{array}{cc}
\frac{\partial^{2}}{v_{0}(\mathbf{x})^{2} \partial t^{2}}-\nabla^{2} & -\frac{m(\mathbf{x}) \partial^{2}}{v_{0}(\mathbf{x})^{2} \partial t^{2}} \\
0 & \frac{\partial^{2}}{v_{0}(\mathbf{x})^{2} \partial t^{2}}-\nabla^{2}
\end{array}\right)\left(\begin{array}{c}
\widehat{p_{0}}\left(\mathbf{x}, t ; \mathbf{x}_{S}\right) \\
\widehat{\delta p}\left(\mathbf{x}, t ; \mathbf{x}_{S}\right)
\end{array}\right)=\left(\begin{array}{c}
0 \\
\Delta d\left(\mathbf{x}_{R}, t ; \mathbf{x}_{S}\right)
\end{array}\right)
$$

in which we could find that the upper row of $\mathbf{F}^{\mathrm{T}}$ is useless in LSRTM, because the adjoint wavefield $\widehat{\delta p}\left(\mathbf{x}, t ; \mathbf{x}_{S}\right)$ is independent of $\widehat{p_{0}}\left(\mathbf{x}, t ; \mathbf{x}_{S}\right)$. We could get access to $\widehat{\delta p}\left(\mathbf{x}, t ; \mathbf{x}_{S}\right)$ directly by

$$
\left(\frac{\partial^{2}}{v_{0}(\mathbf{x})^{2} \partial t^{2}}-\nabla^{2}\right) \widehat{\delta p}\left(\mathbf{x}, t ; \mathbf{x}_{S}\right)=\Delta d\left(\mathbf{x}_{R}, t ; \mathbf{x}_{S}\right)
$$

As for $\partial \mathbf{F} / \partial \mathbf{m}$, we have

$$
\frac{\partial \mathbf{F}}{\partial \mathbf{m}}=\left(\begin{array}{cc}
0 & 0 \\
-\frac{\partial^{2}}{v_{0}(\mathbf{x})^{2} \partial t^{2}} & 0
\end{array}\right)
$$

Finally we could obtain

$$
\frac{\partial \phi(m)}{\partial m}=\left\langle\left(\begin{array}{cc}
0 & 0 \\
-\frac{\partial^{2}}{v_{0}^{2} \partial t^{2}} & 0
\end{array}\right)\left(\begin{array}{c}
p_{0} \\
\delta p
\end{array}\right),\left(\begin{array}{c}
0 \\
\widehat{\delta p}
\end{array}\right)\right\rangle=\int_{0}^{T}-\frac{\partial^{2} p_{0}}{v_{0}^{2} \partial t^{2}} \widehat{\delta p} d t
$$


When the reflectivity is defined as $m(\mathbf{x})=r(\mathbf{x}) / v_{0}(\mathbf{x})$, the mathematical derivation process is similar except for

$$
\begin{array}{r}
\mathbf{F}=\left(\begin{array}{cc}
\frac{\partial^{2}}{v_{0}(\mathbf{x})^{2} \partial t^{2}}-\nabla^{2} & 0 \\
-\frac{m(\mathbf{x}) \partial}{\partial t} & \frac{\partial^{2}}{v_{0}(\mathbf{x})^{2} \partial t^{2}}-\nabla^{2}
\end{array}\right), \mathbf{F}^{\mathrm{T}}=\left(\begin{array}{cc}
\frac{\partial^{2}}{v_{0}(\mathbf{x})^{2} \partial t^{2}}-\nabla^{2} & -\frac{m(\mathbf{x}) \partial}{\partial t} \\
0 & \frac{\partial^{2}}{v_{0}(\mathbf{x})^{2} \partial t^{2}}-\nabla^{2}
\end{array}\right) \\
\frac{\partial \mathbf{F}}{\partial \mathbf{m}}=\left(\begin{array}{cc}
0 & 0 \\
-\frac{\partial}{\partial t} & 0
\end{array}\right),
\end{array}
$$

Under the $m(\mathbf{x})=r(\mathbf{x}) / v_{0}(\mathbf{x})$ parameterization, the adjoint-state wavefield could still be expressed as

$$
\left(\frac{\partial^{2}}{v_{0}(\mathbf{x})^{2} \partial t^{2}}-\nabla^{2}\right) \widehat{\delta p}\left(\mathbf{x}, t ; \mathbf{x}_{S}\right)=\Delta d\left(\mathbf{x}_{R}, t ; \mathbf{x}_{S}\right)
$$

but the gradient is

$$
\frac{\partial \phi(m)}{\partial m}=\left\langle\left(\begin{array}{cc}
0 & 0 \\
-\frac{\partial}{\partial t} & 0
\end{array}\right)\left(\begin{array}{c}
p_{0} \\
\delta p
\end{array}\right),\left(\begin{array}{c}
0 \\
\widehat{\delta p}
\end{array}\right)\right\rangle=\int_{0}^{T}-\frac{\partial p_{0}}{\partial t} \widehat{\delta p} d t
$$

\section{APPENDIX B}

CODES ON SOURCE WAVEFIELD RECONSTRUCTION USING FSEEK()

The following codes are written in C: 


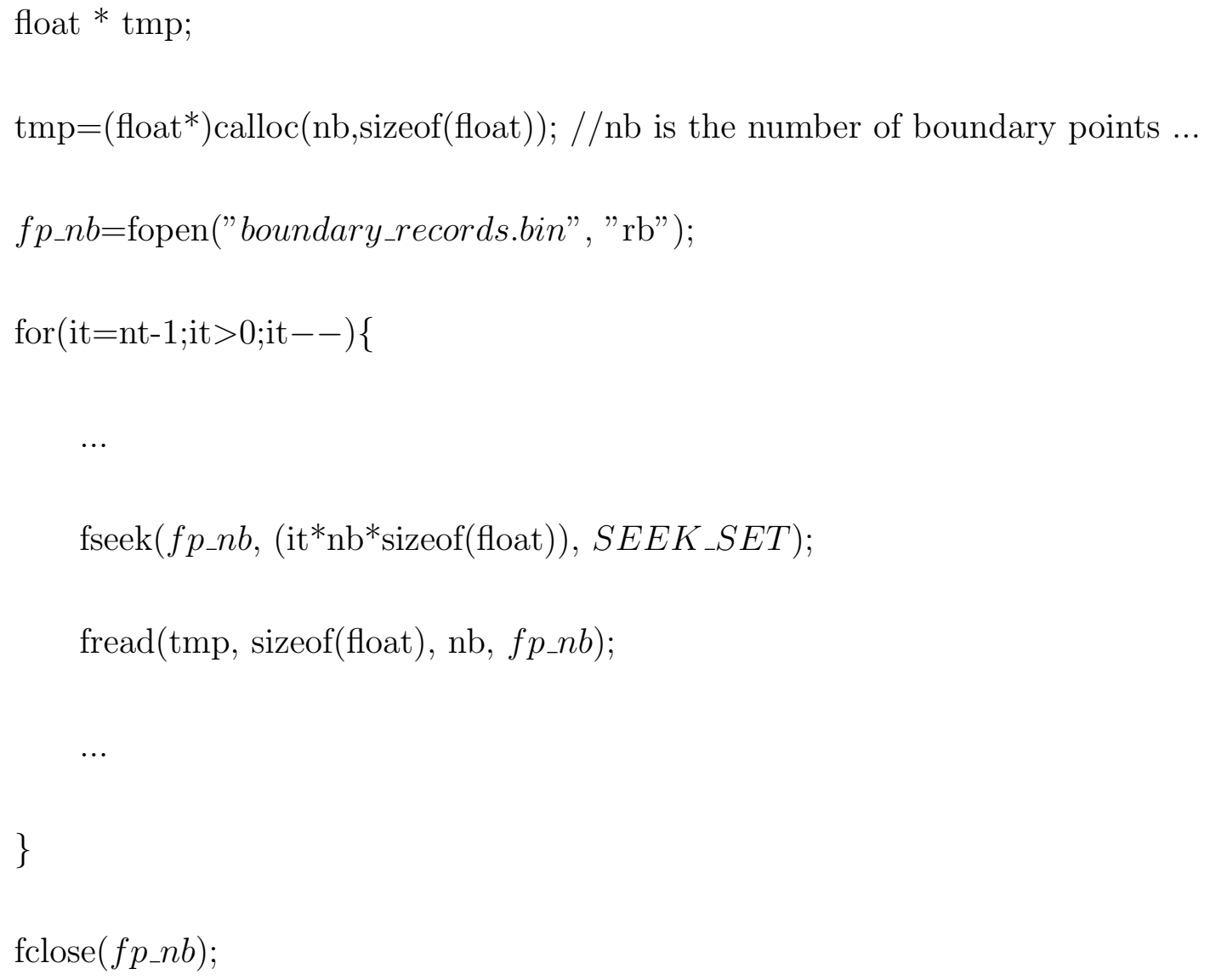




\section{REFERENCES}

Aki, K., and P. G. Richards, 1980, Quantitative seismology, theory and methods i: W. H. Freeman and Co.

Claerbout, J. F., 1992, Earth soundings analysis: Processing versus inversion: Blackwell Science.

Clapp, M. L., R. G. Clapp, and B. L. Biondi, 2005, Regularized least-squares inversion for 3D subsalt imaging: 75rd Annual International Meeting, SEG, Expanded Abstracts, 1814-1817.

Clément, F., G. Chavent, and S. Gómez, 2001, Migration-based traveltime waveform inversion of 2-D simple structures: A synthetic example: Geophysics, 66, 845-860.

Dai, W., and G. T. Schuster, 2013, Plane-wave least-squares reverse-time migration: Geophysics, 78, S165-S177.

Dutta, G., and G. T. Schuster, 2014, Attenuation compensation for least-squares reverse time migration using the viscoacoustic-wave equation: Geophysics, 79, S251-S262.

Kuehl, H., and M. Sacchi, 2002, Robust AVP estimation using least-squares waveequation migration: 72rd Annual International Meeting, SEG, Expanded Abstracts, 21, 281-284.

Lailly, P., 1983, The seismic inverse problem as a sequence of before stack migrations: Conference on inverse scattering, theory and application: Society for Industrial and Applied Mathematics, 206-220.

Liu, Q., 2016, Improving the gradient in least-squares reverse time migration: Journal of Geophysics and Engineering, 13, 172-180. 
Liu, Q., and D. Peter, 2018, One-step data-domain least-squares reverse-time migration: Geophysics, 83, 1-46.

Liu, Q., J. Zhang, and H. Gao, 2017a, Reverse-time migration from rugged topography using irregular, unstructured mesh: Geophysical Prospecting, 65, 453-466.

Liu, Q., J. Zhang, and Z. Hao, 2016a, Eliminating the redundant source effects from the cross-correlation reverse-time migration using a modified stabilized division: Computers and Geosciences, 92, 49-57.

Liu, S., X. Li, W. Wang, and T. Zhu, 2015, Source wavefield reconstruction using a linear combination of the boundary wavefield in reverse time migration: Geophysics, 80, S203-S212.

Liu, X., Y. Liu, H. Lu, H. Hao, and M. Khan, 2017b, Prestack correlative leastsquares reverse time migration: Geophysics, 82, S159-S172.

Liu, Y., J. Teng, X. Tao, Z. Bai, H. Lan, and J. Badal, 2016b, An efficient steplength formula for correlative least-squares reverse time migration: Geophysics, 81, S221-S238.

Nemeth, T., C. Wu, and G. T. Schuster, 1999, Least-squares migration of incomplete reflection data: Geophysics, 64, 208-221.

Plessix, R. E., and W. A. Mulder, 2004, Frequency-domain finite-difference amplitude-preserving migration: Geophysical Journal International, 157, 975987.

Schuster, G. T., 1993, Least-squares cross-well migration: 63rd Annual International Meeting, SEG, Expanded Abstracts, 12, 110-113.

Tan, S., and L. Huang, 2014, Least-squares reverse-time migration with a wavefield- 
separation imaging condition and updated source wavefields: Geophysics, 79, S195-S205.

Tarantola, A., 1984, Inversion of seismic reflection data in the acoustic approximation: Geophysics, 49, 1259-1266.

Tromp, J., C. Tape, and Q. Liu, 2005, Seismic tomography, adjoint methods, time reversal and banana-doughnut kernels: Geophysical Journal International, 160, $195-216$.

Virieux, J., 1986, P-SV wave propagation in heterogeneous media: velocity-stress finite-difference method: Geophysics, 51, 889-901.

Virieux, J., and S. Operto, 2009, An overview of full-waveform inversion in exploration geophysics: Geophysics, 74, WCC1-WCC26.

Wang, J., H. Kuehl, and M. D. Sacchi, 2005, High-resolution wave-equation AVA imaging: Algorithm and tests with a data set from the Western Canadian Sedimentary Basin: Geophysics, 70, S91-S99.

Wong, M., B. L. Biondi, and S. Ronen, 2015, Imaging with primaries and freesurface multiples by joint least-squares reverse time migration: Geophysics, $\mathbf{8 0}$, S223-S235.

Wu, S., Y. Wang, Y. Zheng, and X. Chang, 2015, Limited-memory BFGS based least-squares pre-stack Kirchhoff depth migration: Geophysical Journal International, 202, 738-747.

Yang, J., and H. Zhu, 2018, Time-domain least-squares Gaussian beam migration with L1 regularization: 88th Annual International Meeting, SEG, Expanded Abstracts, 4266-4270. 
Yang, J., H. Zhu, G. Mcmechan, and Y. Yue, 2018, Time-domain least-squares migration using the gaussian beam summation method: Geophysical Journal International, 214, 548-572.

Yao, G., 2016, Least squares reverse-time migration in a matrix-based formulation: Geophysical Prospecting, 64, 611-621.

Yuan, M., J. Huang, W. Liao, and F. Jiang, 2015, Least-squares gaussian beam migration: Geophysics, 81, S87-S100.

Zhang, Y., L. Duan, and Y. Xie, 2015, A stable and practical implementation of least-squares reverse time migration: Geophysics, 80, V23-V31. 


\section{LIST OF FIGURES}

1 1D illustration about how to get normal-incidence reflection coefficient from velocity perturbation. (a) True velocity model. (b) smoothed velocity model of (a) by a 400m Gaussian smoother. (c) velocity perturbation obtained by (a) minus (b). (d) Normal incidence reflection coefficient model

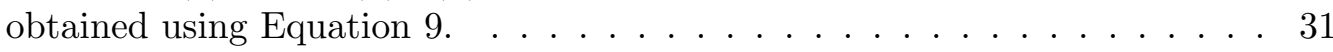

2 Illustration about how to fetch values from a large file on this disk using fseek. In this case only a small vector of $\mathrm{Nb}^{*} 1$ is required. The memory cost will be reduced a lot. . . . . . . . . . . . . . . . . . . . . . 32

3 (a) The velocity model; (b) $m_{\delta v}(\mathbf{x})$ related RTM imaging profile; (c) $m_{\delta v}(\mathbf{x})$ related LSRTM inverted image; (d) $m_{r}(\mathbf{x})$ related RTM imaging profile; (e) $m_{r}(\mathbf{x})$ related LSRTM inverted image. . . . . . . . . . . . . . 33

4 (a) Extracted well-logs from Figure 3c at location $1.5 \mathrm{~km}$; (b) Extracted

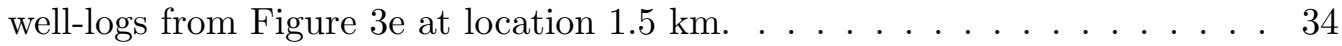

5 (a) True Marmousi velocity model. (b) Background velocity model. . . . . . 35

6 (a) True Marmousi velocity perturbation related reflectivity model $m_{\delta v}(\mathbf{x})$.

(b) RTM imaging profile. (c) $m_{\delta v}(\mathbf{x})$ related LSRTM inverted image. . . . . 36

7 (a) True Marmousi normal-incidence reflection coefficients related reflectivity model $m_{r}(\mathbf{x})$. (b) RTM imaging profile. (c) $m_{r}(\mathbf{x})$ related LSRTM inverted image. . . . . . . . . . . . . . . 37

8 Detailed zoom-views from Figures $6 \mathrm{~b}$ and $6 \mathrm{c}$, Figures $7 \mathrm{~b}$ and $7 \mathrm{c}$ (as indicated by the black dashed boxes), respectively. Top of the Figures 8a and $8 \mathrm{~b}$ is the velocity perturbation related reflectivity model from Figure $6 \mathrm{~b}$, bottom is the corresponding LSRTM inverted image from Figure 6c. Top of the Figures $8 \mathrm{c}$ and $8 \mathrm{~d}$ is the normal-incidence reflection coefficients related reflectivity model from Figure 7b, bottom is the corresponding LSRTM inverted image from Figure 7 c. . . . . . . . . . . . . . . 38

9 Comparison between the amplitude spectra at location $6.5 \mathrm{~km}$ of two LSRTMs under different reflectivity definitions. The traces of $\mathrm{a}$ and $\mathrm{b}$ are extracted from Figures $6 \mathrm{~b}$ and $6 \mathrm{c}$, respectively. The traces of $\mathrm{c}$ and $\mathrm{d}$ are extracted from Figures $7 \mathrm{~b}$ and $7 \mathrm{c}$, respectively. . . . . . . . . . . . 39

10 Comparison of two convergence curves of two LSRTMs under different reflectivity definitions. The curve of $m_{\delta v}(\mathbf{x})$ inversion is in red and that of $m_{r}(\mathbf{x})$ is in blue. The blue line goes down a bit slower than the red one. We explain their difference as follows. The $m_{\delta v}(\mathbf{x})$ related Born modeling has already been an approximation to the full wave-equation modeling, and the $m_{r}(\mathbf{x})$ related Born modeling regarding is a further approximation to the forward modeling. The further approximation means less accuracy in the data prediction. Also, in the $m_{r}(\mathbf{x})$ approximation, we neglect the AVA behaviors, which should be considered in the further research. . . . . . . . 40 
11 Comparison of traces from inverted results (solid lines) and true reflectivity models (dash lines) at location $4 \mathrm{~km}$, respectively. The comparison group in (a) is extracted from Figures 6a and 6c, respectively. The comparison group in (b) is extracted from Figures 7a and 7c, respectively. We observe that in both cases the inverted results match well with the corresponding defined reflectivity models. The inverted result in (b) has more high-wavenumber components because its reflectivity definition, in which each reflector is defined as a delta function. . . . . . . . . . . . . . . . . 

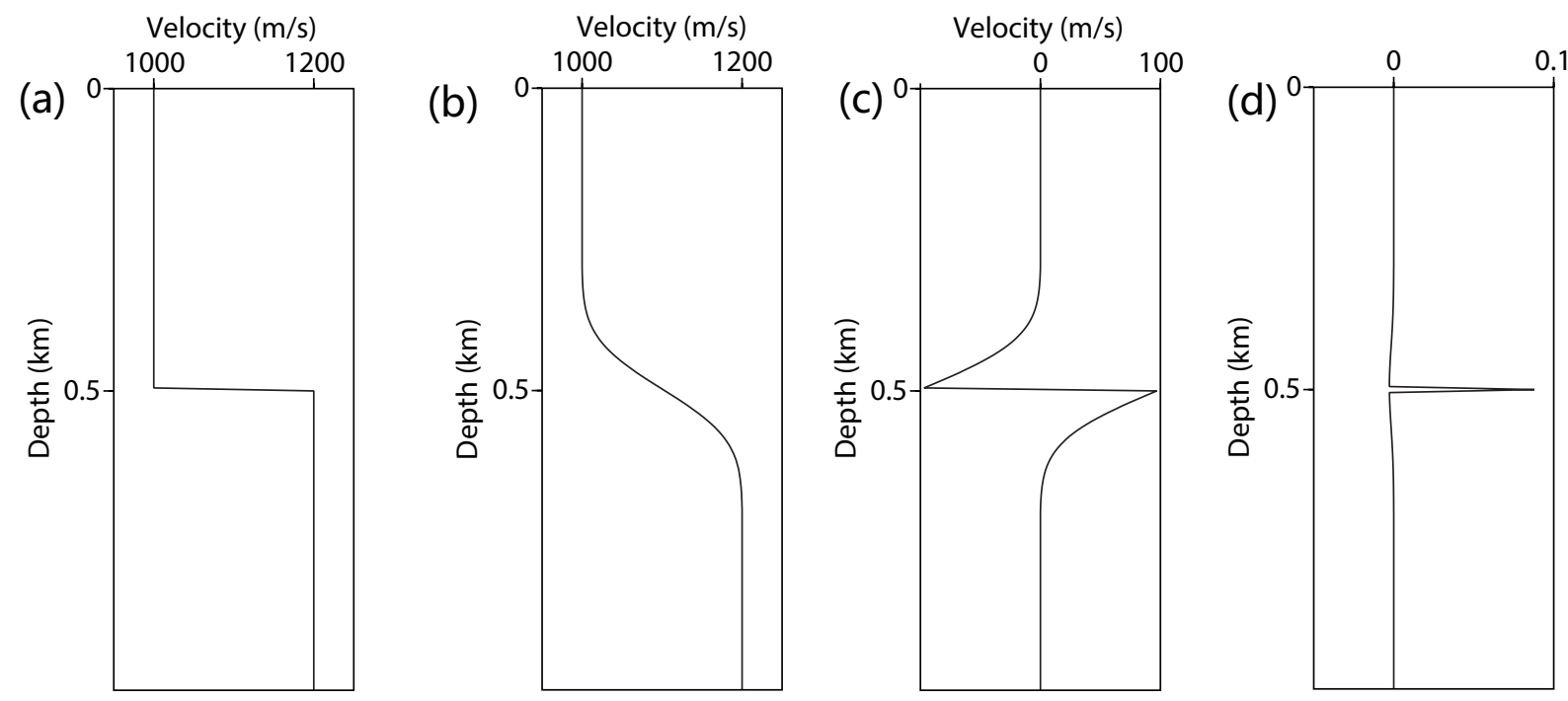

Figure 1: 1D illustration about how to get normal-incidence reflection coefficient from velocity perturbation. (a) True velocity model. (b) smoothed velocity model of (a) by a 400m Gaussian smoother. (c) velocity perturbation obtained by (a) minus (b). (d) Normal incidence reflection coefficient model obtained using Equation 9. 


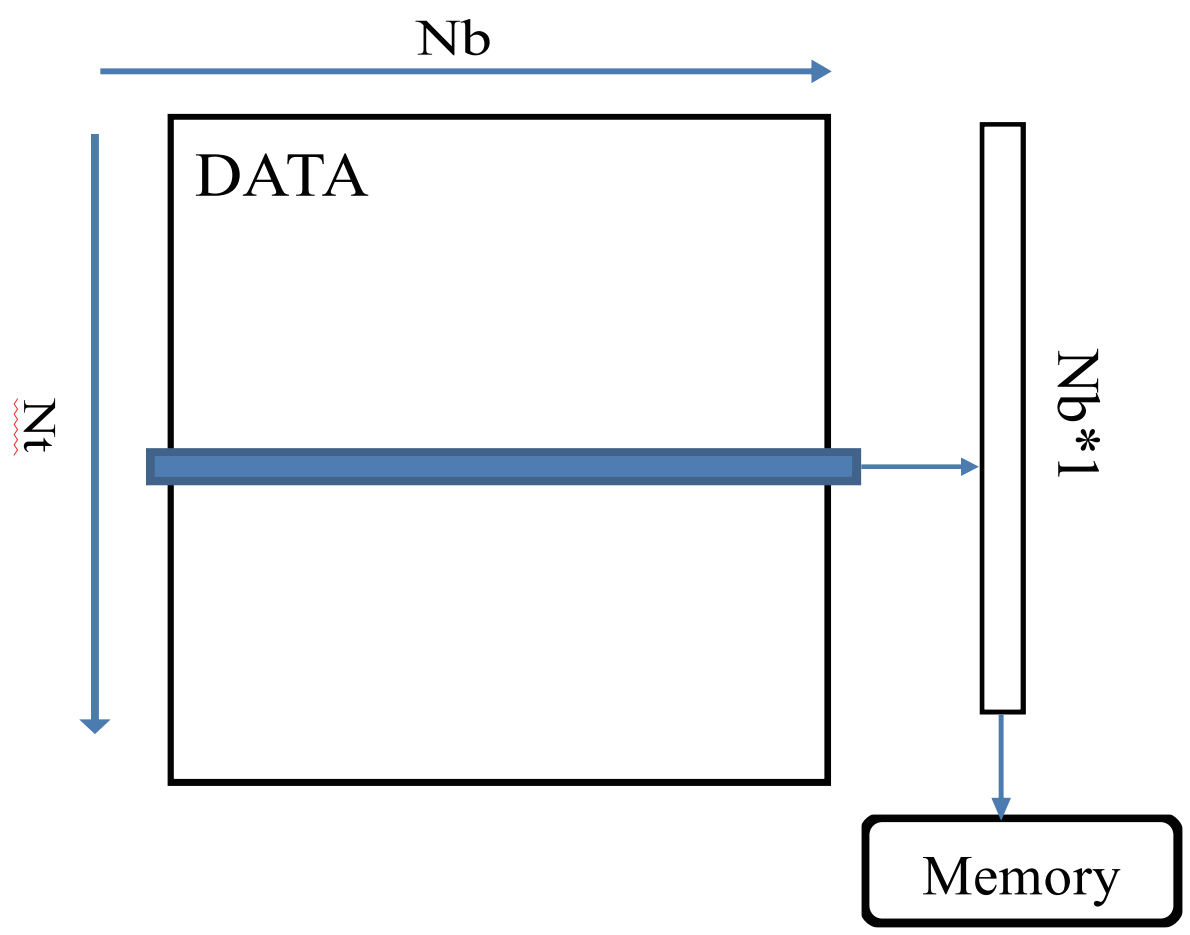

Figure 2: Illustration about how to fetch values from a large file on this disk using fseek. In this case only a small vector of $\mathrm{Nb}^{*} 1$ is required. The memory cost will be reduced a lot. 


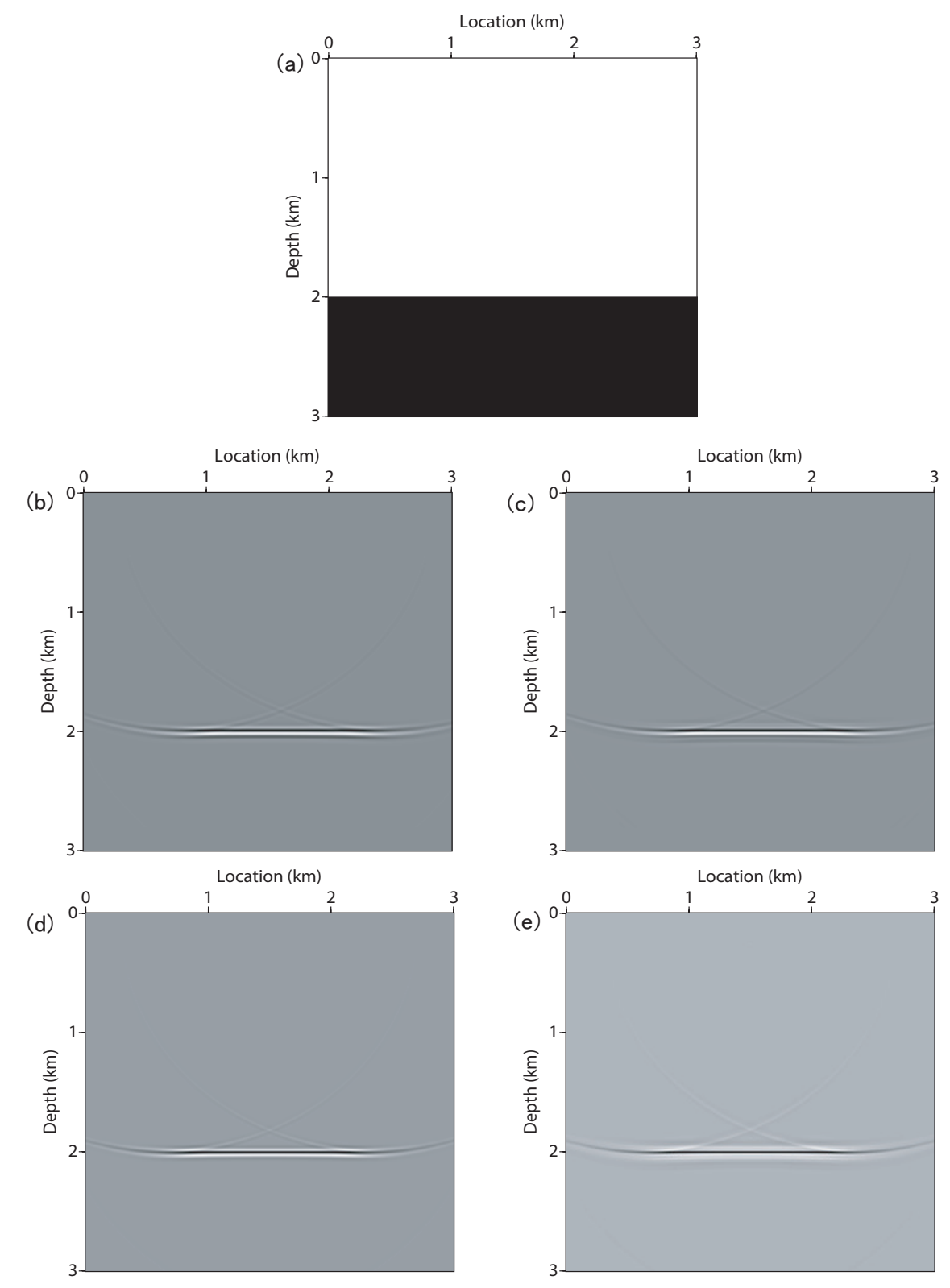

Figure 3: (a) The velocity model; (b) $m_{\delta v}(\mathbf{x})$ related RTM imaging profile; (c) $m_{\delta v}(\mathbf{x})$ related LSRTM inverted image; (d) $m_{r}(\mathbf{x})$ related RTM imaging profile; (e) $m_{r}(\mathbf{x})$ related LSRTM inverted image. 

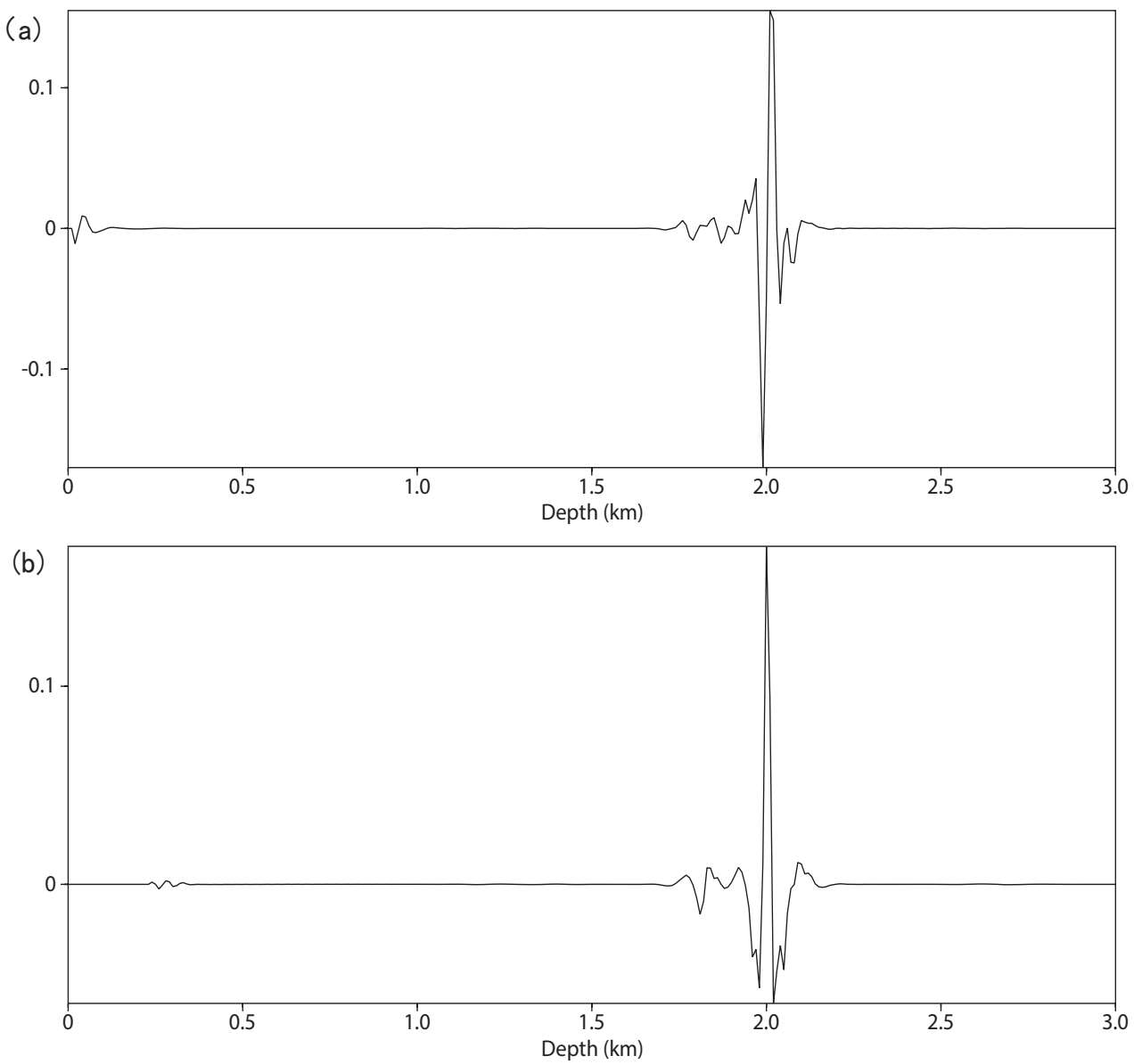

Figure 4: (a) Extracted well-logs from Figure 3c at location $1.5 \mathrm{~km}$; (b) Extracted well-logs from Figure $3 \mathrm{e}$ at location $1.5 \mathrm{~km}$. 

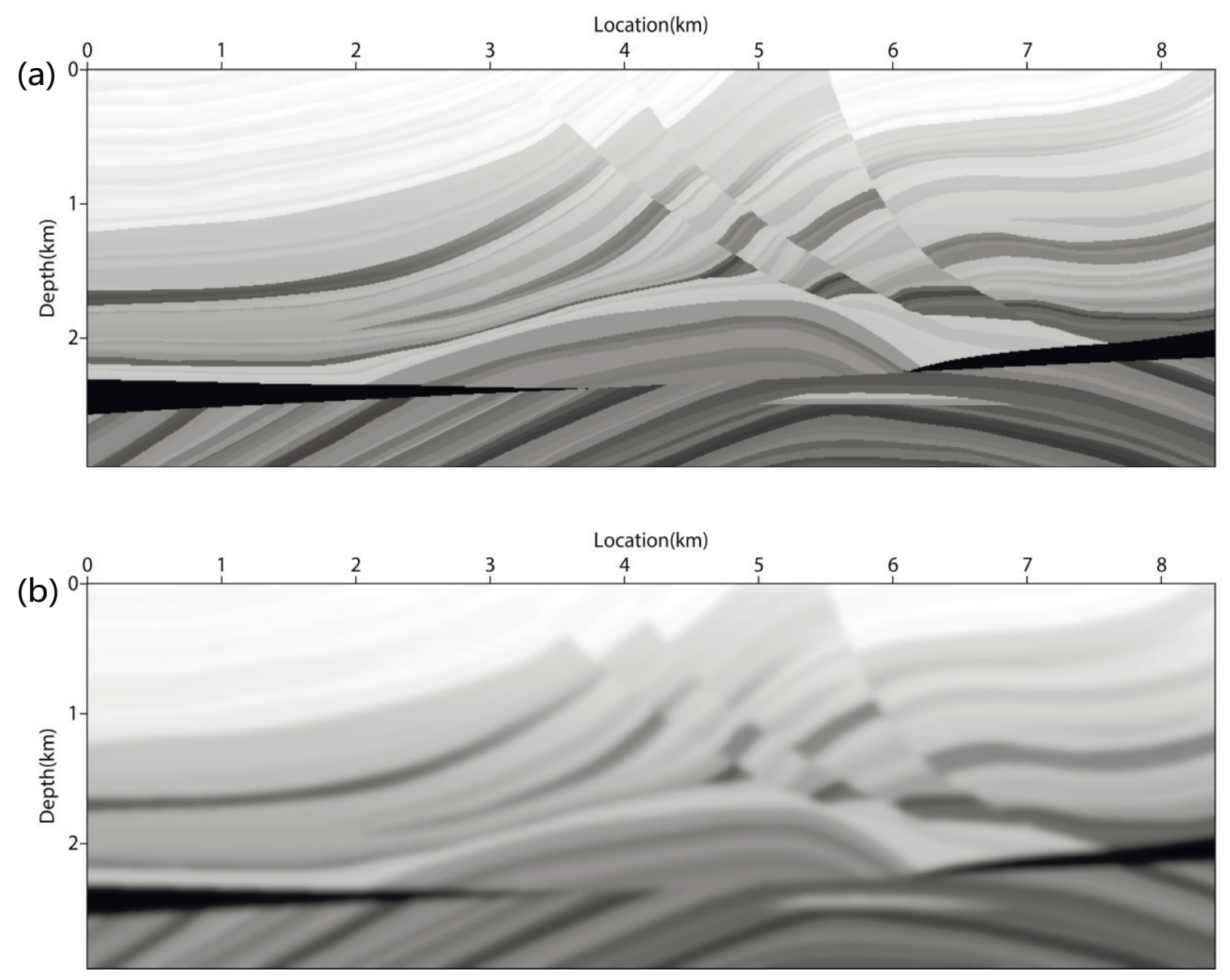

Figure 5: (a) True Marmousi velocity model. (b) Background velocity model. 

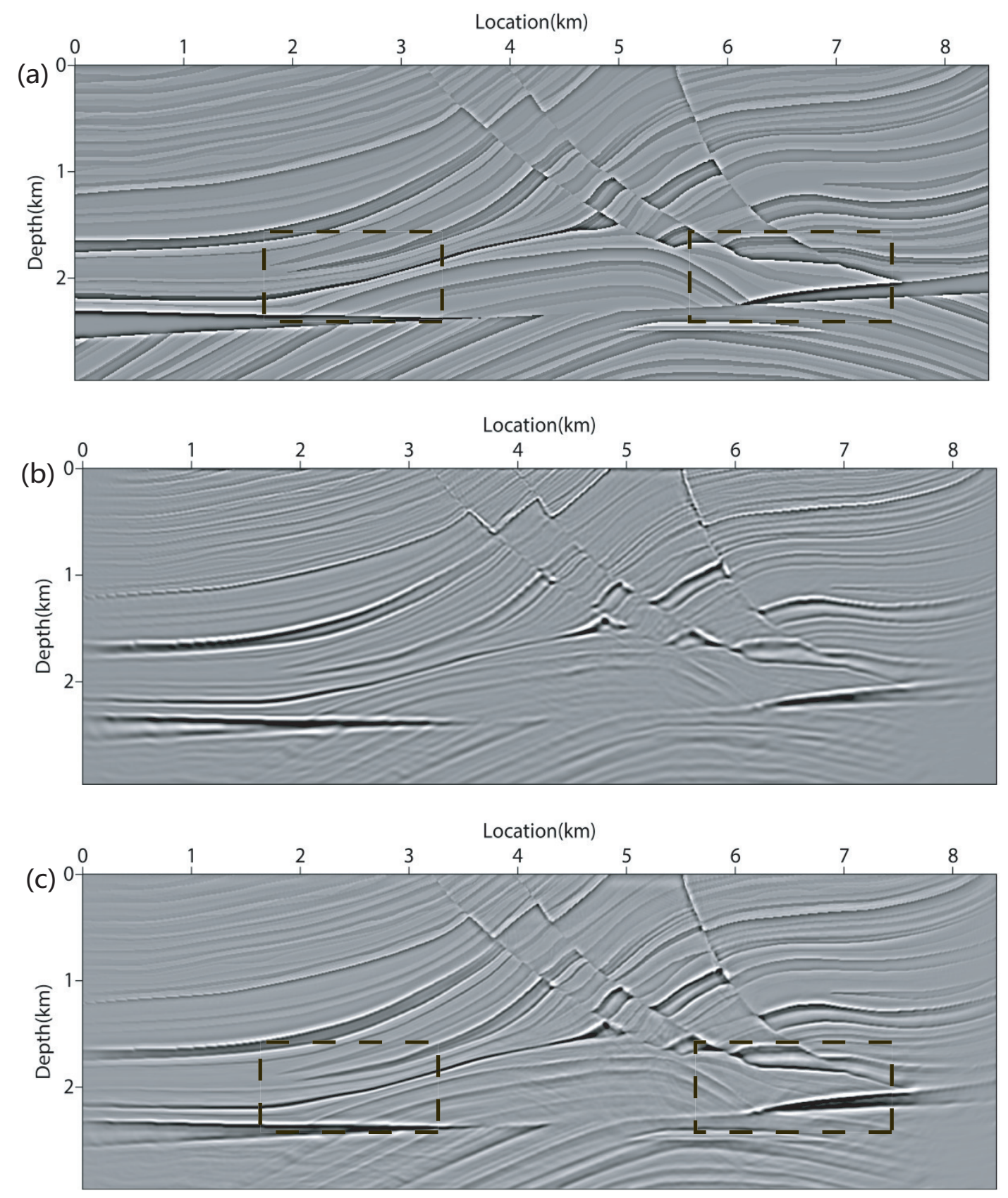

Figure 6: (a) True Marmousi velocity perturbation related reflectivity model $m_{\delta v}(\mathbf{x})$. (b) RTM imaging profile. (c) $m_{\delta v}(\mathbf{x})$ related LSRTM inverted image. 

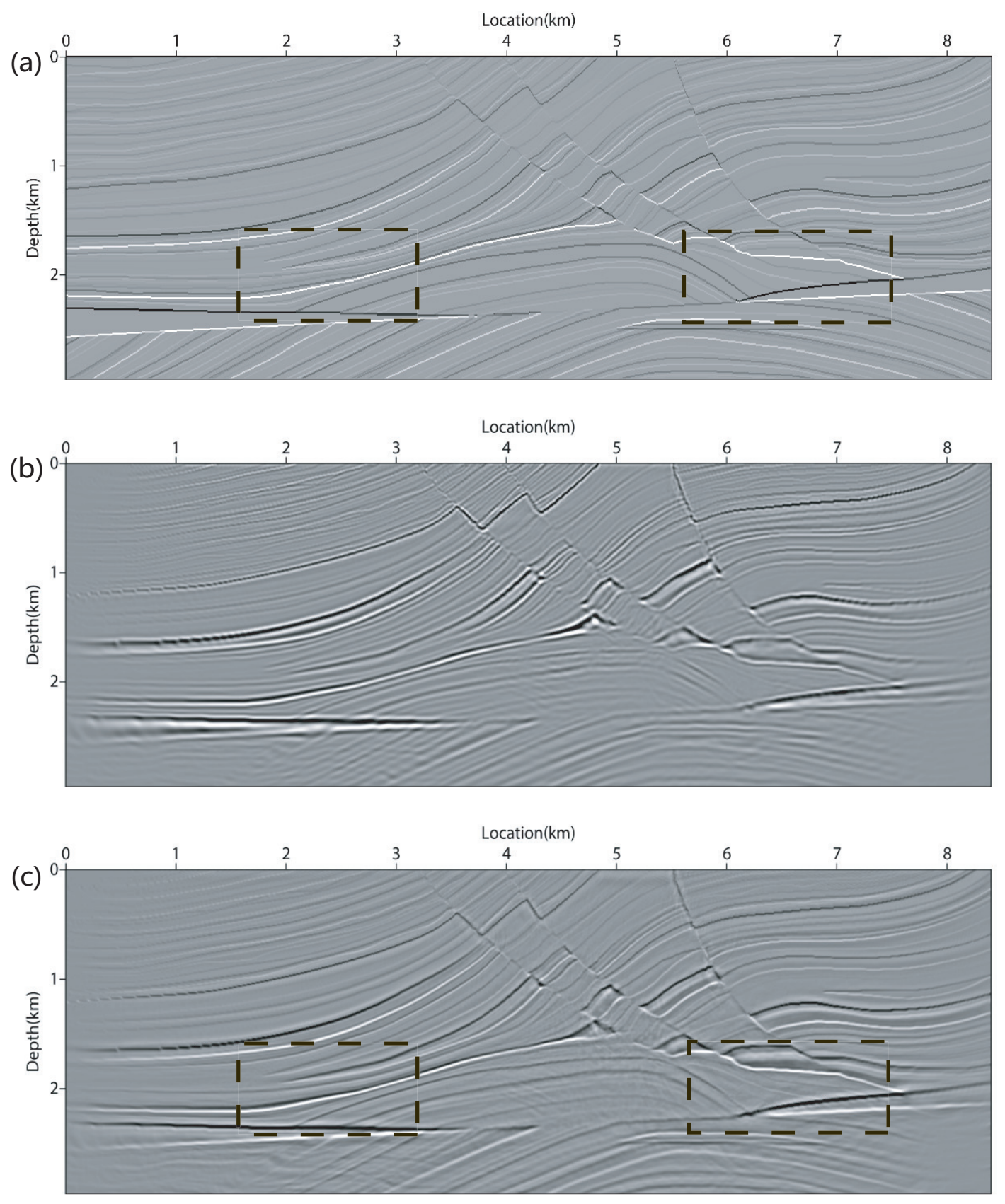

Figure 7: (a) True Marmousi normal-incidence reflection coefficients related reflectivity model $m_{r}(\mathbf{x})$. (b) RTM imaging profile. (c) $m_{r}(\mathbf{x})$ related LSRTM inverted image. 

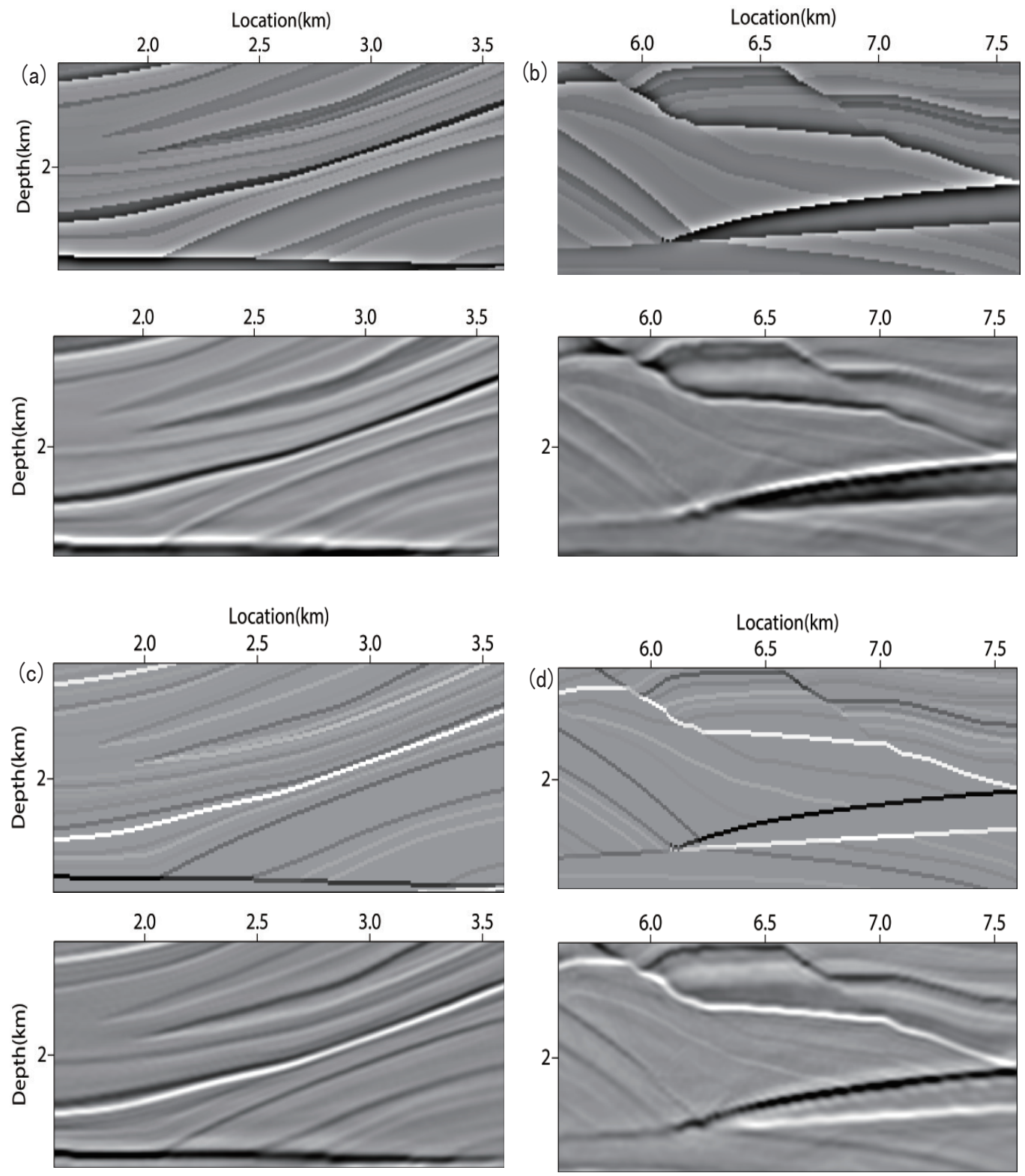

Figure 8: Detailed zoom-views from Figures $6 \mathrm{~b}$ and $6 \mathrm{c}$, Figures $7 \mathrm{~b}$ and $7 \mathrm{c}$ (as indicated by the black dashed boxes), respectively. Top of the Figures $8 \mathrm{a}$ and $8 \mathrm{~b}$ is the velocity perturbation related reflectivity model from Figure $6 \mathrm{~b}$, bottom is the corresponding LSRTM inverted image from Figure 6c. Top of the Figures $8 \mathrm{c}$ and $8 \mathrm{~d}$ is the normal-incidence reflection coefficients related reflectivity model from Figure $7 \mathrm{~b}$, bottom is the corresponding LSRTM inverted image from Figure 7c. 

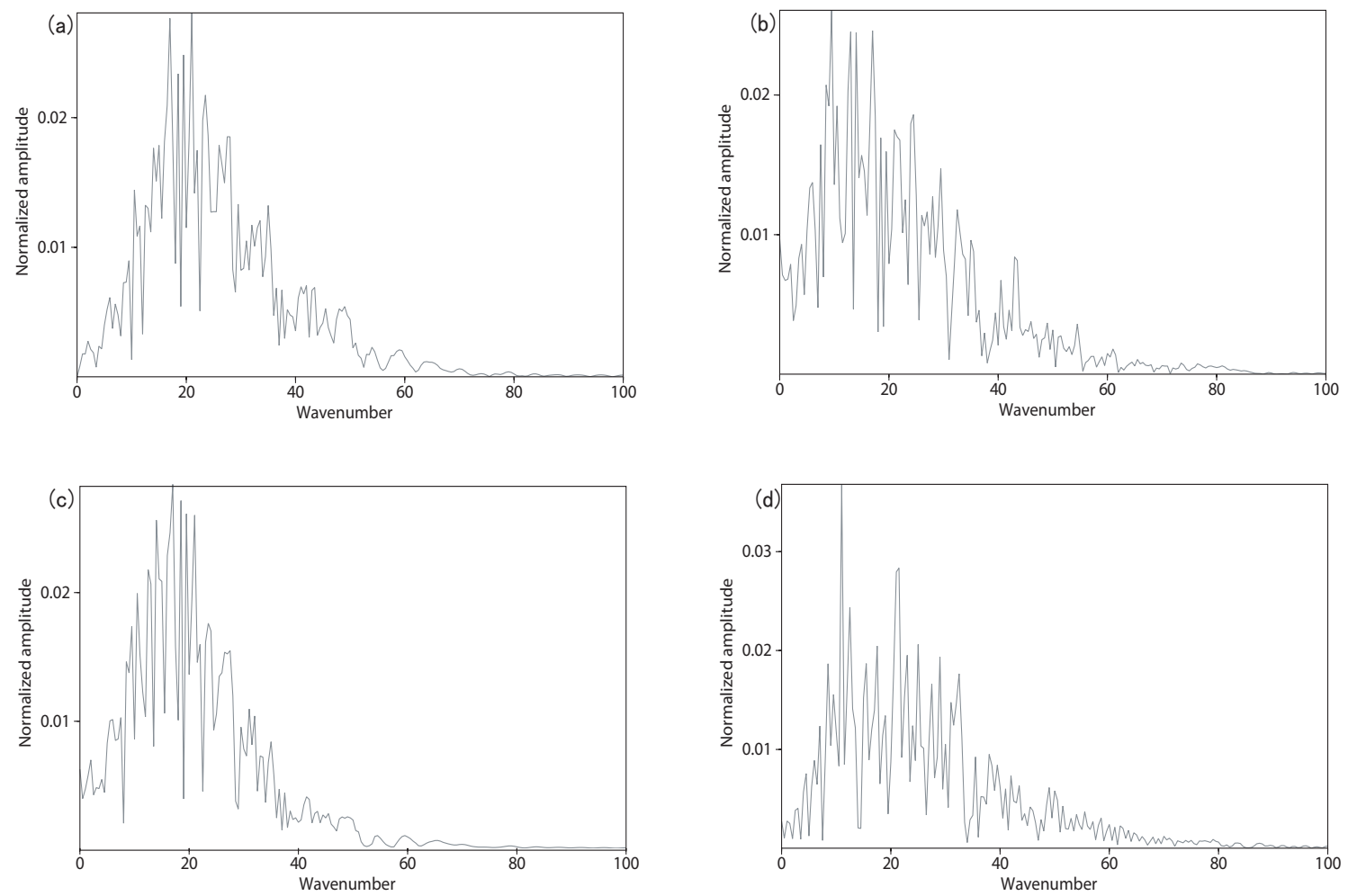

Figure 9: Comparison between the amplitude spectra at location $6.5 \mathrm{~km}$ of two LSRTMs under different reflectivity definitions. The traces of $\mathrm{a}$ and $\mathrm{b}$ are extracted from Figures $6 \mathrm{~b}$ and $6 \mathrm{c}$, respectively. The traces of $\mathrm{c}$ and $\mathrm{d}$ are extracted from Figures $7 \mathrm{~b}$ and $7 \mathrm{c}$, respectively. 


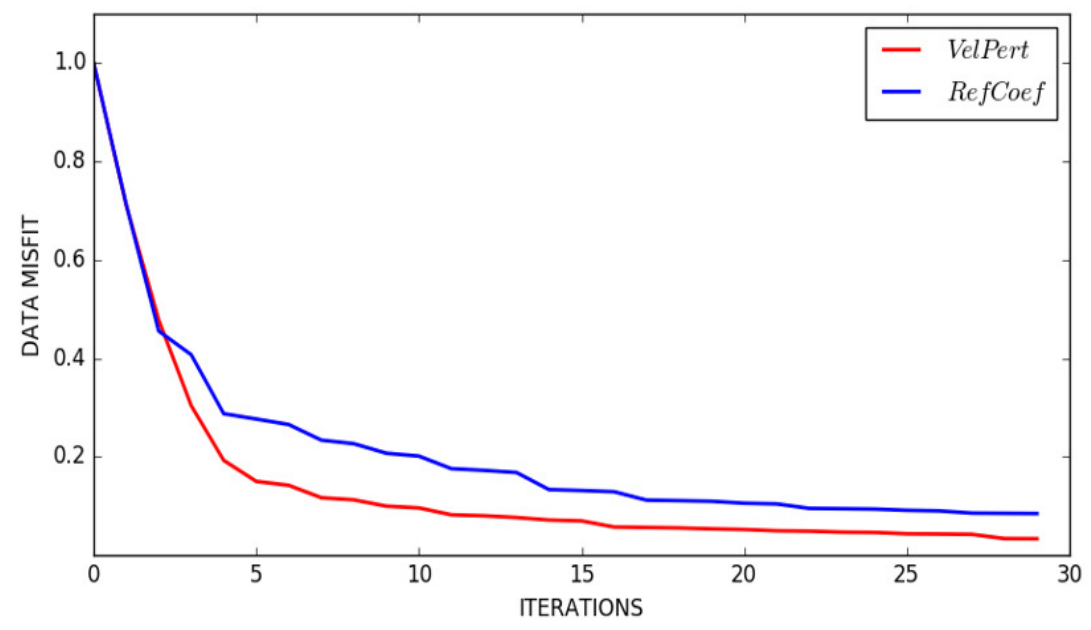

Figure 10: Comparison of two convergence curves of two LSRTMs under different reflectivity definitions. The curve of $m_{\delta v}(\mathbf{x})$ inversion is in red and that of $m_{r}(\mathbf{x})$ is in blue. The blue line goes down a bit slower than the red one. We explain their difference as follows. The $m_{\delta v}(\mathbf{x})$ related Born modeling has already been an approximation to the full wave-equation modeling, and the $m_{r}(\mathbf{x})$ related Born modeling regarding is a further approximation to the forward modeling. The further approximation means less accuracy in the data prediction. Also, in the $m_{r}(\mathbf{x})$ approximation, we neglect the AVA behaviors, which should be considered in the further research. 

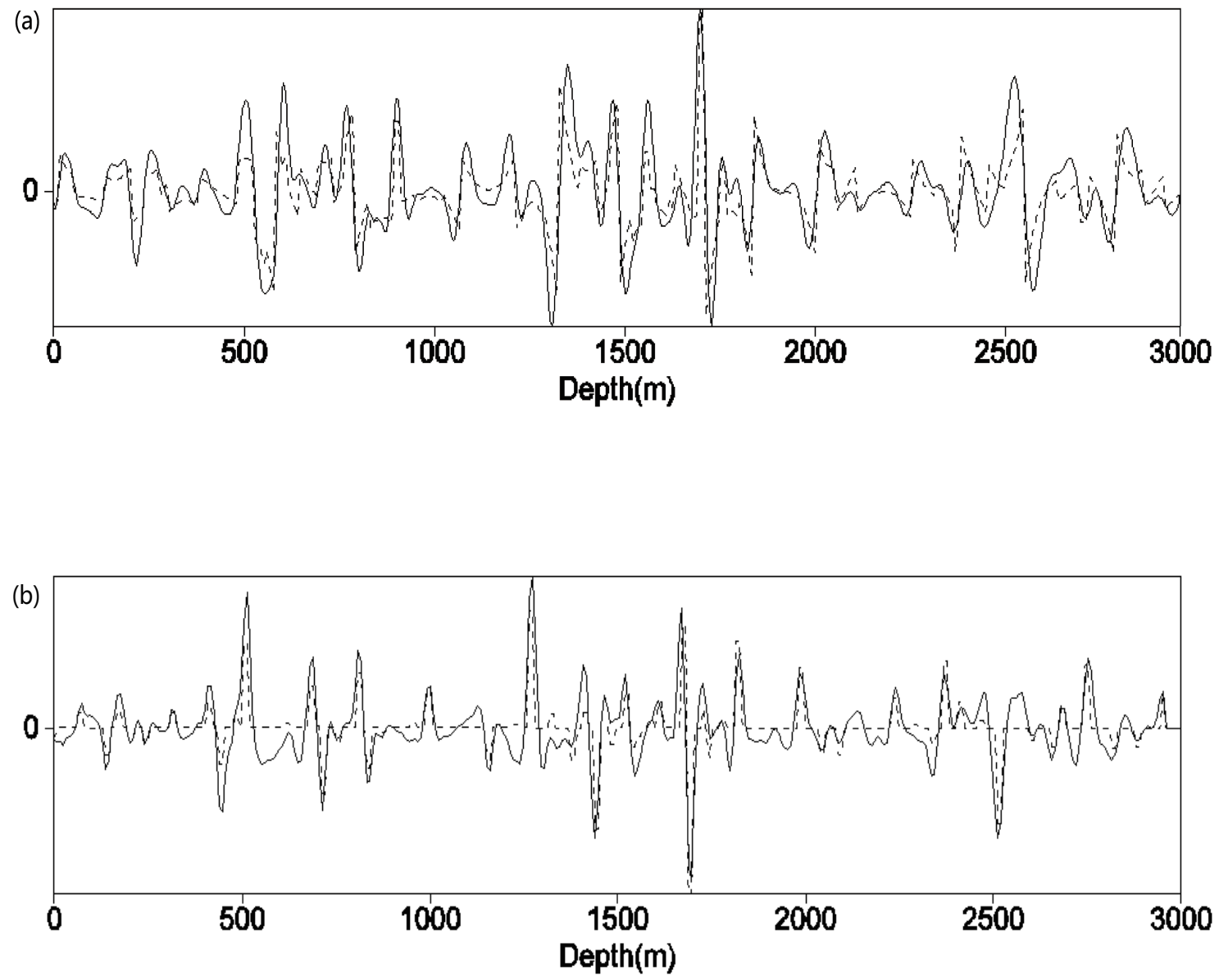

Figure 11: Comparison of traces from inverted results (solid lines) and true reflectivity models (dash lines) at location $4 \mathrm{~km}$, respectively. The comparison group in (a) is extracted from Figures 6a and 6c, respectively. The comparison group in (b) is extracted from Figures $7 \mathrm{a}$ and $7 \mathrm{c}$, respectively. We observe that in both cases the inverted results match well with the corresponding defined reflectivity models. The inverted result in (b) has more high-wavenumber components because its reflectivity definition, in which each reflector is defined as a delta function. 